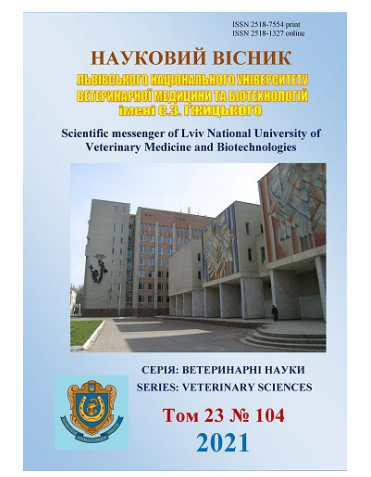

\author{
Науковий вісник Дьвівського національного університету \\ ветеринарної медицини та біотехнологій імені С.3. Гжицького. \\ Серія: Ветеринарні науки
}

\author{
Scientific Messenger of Lviv National University \\ of Veterinary Medicine and Biotechnologies. \\ Series: Veterinary sciences
}

doi: 10.32718/nvlvet10405

https://nvlvet.com.ua/index.php/journal

UDC 623.4:633.854.78

\title{
Research Progress on the pathogenic mechanism of Streptococcus suis 2
}

\author{
Mingcheng Liu ${ }^{1,2}$, Xiaojing $\mathrm{Xia}^{2}$, Xingyou Liu ${ }^{3}$, Oksana Kasianenko ${ }^{1}$ \\ ${ }^{1}$ Faculty of Veterinary Medicine, Sumy National Agrarian University, Sumy, Ukraine \\ ${ }^{2}$ Henan Institute of Science and technology, Xin xiang, China \\ ${ }^{3}$ Xinxiang University, Xin xiang, China
}

Article info

Received 14.09.2021

Received in revised form 18.10 .2021

Accepted 19.10.2021

Faculty of Veterinary Medicine, Sumy National Agrarian University, Herasima Kondratieva Str., 160, Sumy, 40021, Ukraine. Tel.: +38-096-069-09-02 E-mail: oksana_kasjanenko@ukr.net

Henan Institute of Science and technology, Xin xiang, 453003, China.

Xinxiang University, Xin xiang, 453003, China.

\begin{abstract}
Liu, M., Xia, X., Liu, X., \& Kasianenko, O. (2021). Research Progress on the pathogenic mechanism of Streptococcus suis 2. Scientific Messenger of Lviv National University of Veterinary Medicine and Biotechnologies. Series: Veterinary sciences, 23(104), 30-35. doi: $10.32718 /$ nvlvet10405
\end{abstract}

Streptococcus suis (S.suis) is an important zoonotic pathogen that can cause many diseases in pigs, such as sepsis, arthritis, endocarditis, and meningitis, of which meningitis is the most serious. There are 35 serotypes, and serotype two is the most virulent. At the same time, Streptococcus suis serotype 2(SS2) can also infect humans, causing severe public health problems. Although SS2 has attracted significant attention worldwide, the research on its pathogenesis is still limited. The adhesion of pathogenic bacteria to the surface of host cells or tissues and its subsequent invasion and diffusion are the critical steps of pathogenic bacteria. Moreover, the interaction between pathogen and host is involved in these processes. Therefore, to study the pathogenic mechanism of pathogenic bacteria is to study the interaction between pathogenic bacteria and host. This paper described several common virulence factors, such as CPS, SLY, $M R P, E F, S A O, S r t, F B P S, S a d P$, and Eno. Under the actions of virulence factors, SS2 adheres and colonizes to the mucosal and epithelial surface of host cells. Then SS2 invades into deeper tissues and bloodstream. If SS2 in the blood does not cause fatal sepsis, It can go to the third stage. The third stage is to cross the BBB and access the CNS and ultimately causes meningitis. During pathogenesis, SS2 interacts with multiple host cells, such as neutrophils, macrophages, epithelial cells, and microvascular endothelial cells to evade the innate or adaptive immunity of the host.

Key words: Research, pathogenic mechanism, Streptococcus suis 2.

\section{Introduction}

Streptococcus suis is an important bacterial pathogen of swine disease, which often causes significant losses to the global pig industry. S.suis can be divided into 35 serotypes (type 1-34, type 1/2) according to the antigenicity of its capsular polysaccharide, among which serotype 2 of S.suis is the most virulent and the most widespread. Infected pigs often develop meningitis, arthritis, endocarditis, and septicemia, and meningitis is the most significant, showing fibrosis and edema of the meninges (Hlebowicz et al., 2019). In recent years, the incidence of SS2 meningitis has increased significantly.

SS2 is also an important zoonotic pathogen. In addition to SS2, types $1,4,14$, and 16 can also infect humans and cause death (Agoston et al., 2020). Human cases of streptococcal SS2 infection have been reported in many countries, with China having two outbreaks of streptococ- cal type 2 infection in 1998 and 2005 (Du et al., 2017). As a result, SS2 has caused serious public health problems globally.

Although there are many different pathogenic serotypes, the virulence-related factors of SS2 are almost exclusively studied. At present, the virulence factors of SS2 have mainly focused on cell wall-related proteins and secretory factors (Haas \& Grenier, 2018). Multiple virulence factors have been reported for SS2, including muramidase-released protein, extracellular factor, capsule, fibronectin-binding protein, suilysin, sortase, autolysin, IgA hydrolase, and superoxide. These virulence factors are mainly involved in adhesion, antiphagocytosis, invasion, and activation of inflammatory pathways ( Tang et al., 2012; Feng et al., 2014). 


\section{Major virulence factor}

Capsular polysaccharide (CPS)

CPS is one of the typing signs of SS2, and it is also an essential protein and virulence factor for SS2 to resist phagocytosis by macrophages. The capsule is mainly composed of $\mathrm{N}$-acetylneuraminine, galactose, glucose, rhamnose, and N-acetylglucosamine (Segura et al., 2017). Compared with the wild strain, the resistance of CPS deletion strain to phagocytosis and killing of macrophages was significantly reduced. CPS contributed to the resistance of the SS2 strain to the phagocytosis-mediated killing and played a significant role in the evasion of trapping and further killing by the neutrophil extracellular traps (NETs ) (Zhao et al., 2015).

\section{Suilysin(Sly)}

Sly is a cholesterol-dependent pore-forming cytotoxin, found in most virulent strains of SS2, expressed in the medium, secreted in the supernatant of the medium. The mature Sly protein has a molecular weight of approximately $54 \mathrm{kDa}$. Sly will lose its hemolytic activity after oxidation, and cholesterol can also inhibit its hemolytic activity (He et al., 2014). Sly can lyse and damage a variety of cells and plays a vital role in SS2 invading the central system and destroying the BBB. In addition, Sly can induce the changes of host cytoskeleton ( $\mathrm{Lv}$ et al., 2014) and the release of proinflammatory and immunomodulatory cytokines and chemokines, such as interlukin6(IL-6), interlukin-8(IL-8), tumor necrosis factor- $\alpha$ (TNF$\alpha$ ), and interlukin-8(IL-10) (Vötsch et al., 2019).

Muramidase-released protein (MRP) and Extracellular factor (EF)

MPR is a $136 \mathrm{kDa}$ cell wall protein. MRP is released after the action of bacterial enzymes on virulent $S S 2$. The research found that MRP may play a significant role in crossing the $\mathrm{BBB}$ and binding to the host cell, and the variable regions of MRP are involved in virulence ( $\mathrm{Li}$ et al., 2017).

$\mathrm{EF}$ is an extracellular protein with a molecular weight of approximately $110 \mathrm{kDa}$ encoded by the epf gene. It is a secreted protein and can only be detected in the supernatant of the culture medium. MRP and EF are related to the virulence of SS2. However, natural strains lacking MRP and EF are still pathogenic. It may be that the strains expressing MRP and EF are more virulent than the strains without MRP and EF (Guo et al., 2021). Both MRP and EF can be expressed in SS2 strains isolated from diseased pigs in China, while the non-pathogenic strainS did not express MRP and EF (Wang et al., 2017).

\section{Fibronectin- and fibrinogen-binding protein (FBPS)}

FBPS is a bacterial surface protein that exists in various serotypes. FBPS are composed of two domains with unique folds. The C-terminus of FBPS binds to the host cell by fibronectin. Moreover, the N-terminus is attached to the bacterial surface, promoting the adhesion of SS2 to host cells (Xia et al., 2019). Compared with the wild strain, FBPS deletion significantly reduced the virulence of the strain (Feng et al., 2014). FBPS can also be used as a bacterial factor to activate the signaling pathway through the $\beta 1$ integrin receptor to induce chemokine production (Musyoki et al., 2016).

\section{S. suis adhesin P(SadP)}

SadP can bind Galabiose Gala1-4gal-oligosaccharides (Kouki et al., 2013). SadP can be divided into PN subtypes and PO subtypes based on the specificity of oligosaccharides (Madar Johansson et al., 2020). The PN subtype is found in systemic strains that cause meningitis, while the Po subtype is distributed in the asymptomatic carrier and respiratory strains. Both types of SadP are primarily bound to porcine lung globotriaosylceramide(Gb3). Galabiose-binding SadP was identified as IPNTG-anchored cell wall protein (Kouki et al., 2011).

\section{Enolase (Eno)}

Eno can bind to $40 \mathrm{~S}$ ribosomal protein SA (RPSA) on the surface of porcine brain microvascular endothelial cells leading to activation of intracellular p38/ERK-eIF4E signaling, which promotes intracellular expression of HSPD1 (heat-shock protein family D member 1), and initiation of host-cell apoptosis, and increased BBB permeability facilitating bacterial invasion (Liu et al., 2021a).

\section{Sortase(Srt)}

Sortase (Srt) is an enzyme expressed in gram-positive bacteria. Srt participates in anchoring surface protein to cell-wall envelopes. Surface proteins at the leucineproline-variable amino $\operatorname{acid}(\mathrm{X})$-threonine-glycine (LPXTG) can be cleaved by Srt and the formation of an amide bond between the carboxyl group of threonine(T) and the amino group of cell-wall cross-bridges (Siegel et al., 2017). The deficiency of srtA in S. aureus severely impedes the anchoring of bacterial surface proteins, thereby significantly reducing their adherence and invasion of host epithelial cells resulting in reduced virulence (Zhu et al., 2016).

\section{Surface antigen I (SAO)}

Sao is a surface localization protein conserved in SS2, occurring as one of three alleles and containing the LPVTG motif at the C-terminal (Wang et al., 2020). Sao is present in most SS2 and has classic Gram-positive membrane anchoring proteins, such as MRP and LPXTG membrane anchoring structures (Xia et al., 2019).

\section{Pathogenic process of SS 2}

The brain blood-barrier(BBB) is a vital structure composed of brain microvascular endothelial cells (BMEC), basement membrane, astrocyte protrusions, and pericytes to maintain the stability of the brain environment. Among them, BMEC is the most crucial component of BBB.

The primary function of the BBB is to prevent pathogens and other macromolecular substances from entering the brain tissue through blood circulation. It is a barrier with selective permeability and has an irreplaceable role in maintaining the normal physiological state of the central nervous system(CNS) (Xie et al., 2019). At present, it is believed that there are three main ways for meningitiscausing SS2 to cross the BBB: the cross-cell pathway, the 
"Trojan horse" theory, and the paracellular pathway (Coureuil et al., 2010).

The process of the transcellular pathway mainly includes three steps. The first step is adherence and colonization of the host's mucosal and/or epithelial surface(s) (Dutkiewicz et al., 2018).

This step is performed mainly by the adhesins produced by pathogens. In a study of the early stages of SS2 infection, Segura et al. list 33 specific S. suis proteins or other adhesins capable of adhesion to host mucosal epithelial cells, including known virulence factors: porcine lysosomes, SAO, and enzymes (Segura et al., 2016). By regulating the expression level of Sly, SS2 can perforate the surface of host cells to form an invagenous channel to promote bacterial colonization, induce cell apoptosis or necrosis (Fittipaldi et al., 2012).

The second step is an invasion into deeper tissue and translocation in the bloodstream. SS2 survives and multiplies in the blood, and as the blood circulates to potential colonization sites throughout the body, Eventually, bacteremia and even septicemia of the host are formed (Jenkins et al., 2015).

SS2 can survive in the blood and spread to the central nervous system and other host organs, thanks to the adequate protection of CPS against neutrophils and mononuclear/binuclear macrophages phagocytosis and killing (Dutkiewicz et al., 2018).

The third step is crossing the blood-brain barrier. If $S S 2$ is present in the blood and fails to cause fatal sepsis, it can cross the blood-brain barrier into the central nervous system and cause meningitis. The mechanism of entering the central nervous system is not fully understood.

The last step is inflammation. The clinical manifestations of SS2 infection range from asymptomatic bacteremia to explosive systemic bacteremia to meningitis, a large-scale inflammatory process. Inflammation plays a crucial role in the pathogenesis of systemic and CNS infections caused by SS2 (Gottschalk et al., 2010). The pathogen induces excessive production of proinflammatory cytokines and recruitment and activation of different leukocyte populations, leading to inflammation in the central system (Sun et al., 2020). Central nervous system inflammation can lead to intracranial complications, including cerebral edema, increased intracranial pressure, and cerebrovascular damage (Fittipaldi et al., 2012).

Trojan Horse Theory: $S$. suis can be phagocytosed by monocytes after entering the blood, but it cannot be effectively killed in the blood, so $S$. suis circulates in the blood with monocytes through various barriers (such as the epithelial barrier and blood-brain barrier) and reaches the central nervous system, thus causing damage (Yang et al., 2015).

The paracellular pathway refers to the passage of pathogenic bacteria through the blood-brain barrier through the gap between endothelial cells. Whether tight junction proteins and adhesion proteins between cells are changed is still inconclusive during this process.

\section{Interaction of SS2 with host cells Neutrophils}

Neutrophils are the white blood cells with the most significant number and the most substantial swallowing ability in peripheral blood. The host's primary first defense against SS2 is the phagocytosis of neutrophils. When pathogenic SS2 infects pigs, neutrophils are significantly increased in tonsils, and neutrophils are often infiltrated in the injured sites caused by infection (Seele et al., 2018). Porcine neutrophils do not effectively kill SS2 in the absence of specific antibodies. CPS can protect SS2 from phagocytosis by neutrophils (Pei et al., 2020).

\section{Macrophage}

Tonsil, the gateway for SS2 colonization and invasion, significantly increased macrophages in the crypt epithelium at the initial stage of swine SS2 infection (Auger et al., 2019). SS2 not only attaches to macrophages. Also, resist the swallowing of macrophages. As the concentration of bacteria increases during incubation, it becomes toxic to macrophages.

The interaction between SS2 and macrophages can induce host cells to release proinflammatory cytokines and chemokines. Like tumor necrosis factor- $\alpha(\mathrm{TNF}-\alpha)$, I'll, 1L-6, 1L-8. SS2 with capsule can resist phagocytosis of macrophages. The capsule is a virulence factor for SS2. The capsulated SS2 defines macrophage phagocytosis by inhibiting the activation of PI3K/A kT/PKCa signaling pathways used for phagocytosis (Auger et al., 2018).

\section{Epithelial cell}

The adhesion, invasion, and toxicity of SS2 on different epithelial cell lines A549, Hela, PK15, LLC-PK1, and MDCK were studied (Tenenbaum et al., 2016). The results showed that SS2 could not invade epithelial cells. Adherent epithelial cells were time-dependent and concentration-dependent. CPS seems not to affect adhesion. But lack of CPS. The adhesion rate increased significantly. Other studies have also revealed that SS2 may adhere to epithelial tissue and then act on epithelial tissue through Sly "Perforation", resulting in infection of epithelial tissue (Segura et al., 2017).

\section{Endothelial cell}

In vitro experiments showed that SS2 had an adhesion and invasion effect on BMEC. The surface proteins and cell wall components (mainly teichoic acid) of bacteria interact between SS2 and BMEC (Liu et al., 2021a; Segura, 2020).

Before entering BBB, SS2 can secrete bacterial capsular polysaccharide down-regulation, reduce capsular synthesis and thickness, and expose invasive proteins such as bacterial adhesion virulence factor, lipoteichoic acid, sorting enzyme, and enolase as much as possible, and promote interaction between SS2 and BMEC through Sly protein toxicity. It can combine with extracellular matrix protein, which is suitable for colonizing delicate bacteria (Baums \& Valentin-Weigand, 2009; Haas \& Grenier, 2018).

Adhesion of SS2 to $\mathrm{B}, \mathrm{E}$, and $\mathrm{C}$ results in the production of cytokines and chemokines, increased leukocyte attraction, and BBB permeability. However, the proin- 
flammatory factors in cerebrospinal fluid during meningitis may be derived from microglia, endothelial cells, and migrating white cells. Other cytokines such as TNF- $\alpha$ or IL-1 and SS2 can stimulate the production of proinflammatory cytokines.

It was found that the adhesion of Streptococcus suis to human brain microvascular endothelial cell(HBMEC) could induce HBMEC to produce proinflammatory factors such as IL-6, IL-8, and monocyte chemoattractant protein-1(MCP-1), and the induced expression was proportional to the concentration and action time of bacteria (Zhang et al., 2021).

The adhesion of SS2 to HBMEC may provide the precondition for SS2 to secrete toxic substances and induce the production of cytokines to change the permeability of BBB (Liu et al., 2021b).

\section{Conclusion}

In this paper, several virulence factors of SS2 were described. Each stage of SS2 infection and pathogenesis was comprehensively discussed, the interaction between SS2 and host cells was analyzed, and the role of relevant virulence factors in the interaction was clarified. It provides a reference for further study of SS2 and helps control the disease caused by SS2.

\section{Conflict of interest}

The authors declare that there is no conflict of interest.

\section{References}

Agoston, Z., Terhes, G., Hannauer, P., Gajdacs, M., \& Urban, E. (2020). A fatal case of bacteremia caused by Streptococcus suis in a splenectomized man and a European literature review. Acta Microbiol Immunol Hung, 67(3), 148-155. doi: 10.1556/030.2020.01123.

Auger, J., Dolbec, D., Roy, D., Segura, M., \& Gottschalk, M. (2018). The role of the Streptococcus suis serotype two capsular polysaccharides in the interactions with dendritic cells is strain-dependent but remains critical for virulence. PloS one, 13(7), e0200453. doi: 10.1371/journal.pone.0200453.

Auger, J., Payen, S., Roy, D., Dumesnil, A., Segura, M., \& Gottschalk, M. (2019). Interactions of Streptococcus suis serotype 9 with host cells and role of the capsular polysaccharide: Comparison with serotypes 2 and 14. PloS one, 14(10), e0223864. doi: 10.1371/journal.pone.0223864.

Baums, C., \& Valentin-Weigand, P. (2009). Surfaceassociated and secreted factors of Streptococcus suis in epidemiology, pathogenesis and vaccine development. Animal health research reviews, 10(1), 65-83. doi: 10.1017/s146625230999003x.

Coureuil, M., Lécuyer, H., Scott, M., Boularan, C., Enslen, H., Soyer, M., Mikaty, G., Bourdoulous, S., Nassif, X., \& Marullo, S. (2010). Meningococcus Hijacks a $\beta 2$-adrenoceptor/ $\beta$-Arrestin pathway to cross brain microvasculature endothelium. Cell, 143(7), 1149-1160. doi: 10.1016/j.cell.2010.11.035.
Du, P., Zheng, H., Zhou, J., Lan, R., Ye, C., Jing, H., Jin, D., Cui, Z., Bai, X., Liang, J., Liu, J., Xu, L., Zhang, W., Chen, C., \& Xu, J. (2017). Detection of Multiple Parallel Transmission Outbreak of Streptococcus suis Human Infection by Use of Genome Epidemiology, China, 2005. Emerging infectious diseases, 23(2), 204-211. doi: 10.3201/eid2302.160297.

Dutkiewicz, J., Zając, V., Sroka, J., Wasiński, B., Cisak, E., Sawczyn, A., Kloc, A., \& Wójcik-Fatla, A. (2018). Streptococcus suis: a re-emerging pathogen associated with occupational exposure to pigs or pork products. Part II - Pathogenesis. Annals of agricultural environmental medicine, 25(1), 186-203. doi: 10.26444/aaem/85651.

Feng, Y., Zhang, H., Wu, Z., Wang, S., Cao, M., Hu, D., \& Wang, C. (2014). Streptococcus suis infection: an emerging/reemerging challenge of bacterial infectious diseases? $\quad$ Virulence, 5(4), 477-497. doi: $10.4161 /$ viru. 28595 .

Fittipaldi, N., Segura, M., Grenier, D., \& Gottschalk, M. (2012). Virulence factors involved in the pathogenesis of the infection caused by the swine pathogen and zoonotic agent Streptococcus suis. Future microbiology, 7(2), 259-279. doi: 10.2217/fmb.11.149.

Gottschalk, M., Xu, J., Calzas, C., \& Segura, M. (2010). Streptococcus suis: a new emerging or an old neglected zoonotic pathogen? Future microbiology, 5(3), 371-391. doi: 10.2217/fmb.10.2.

Guo, G., Du, D., Yu, Y., Zhang, Y., Qian, Y., \& Zhang, W. (2021). Pan-genome analysis of Streptococcus suis serotype 2 revealed genomic diversity among strains of different virulence. Transboundary emerging diseases, 68(2), 637-647. doi: 10.1111/tbed.13725.

Haas, B., \& Grenier, D. (2018). Understanding the virulence of Streptococcus suis: A veterinary, medical, and economic challenge. Medecine et maladies infectieuses, 48(3), 159-166. doi: 10.1016/j.medmal.2017.10.001.

He, Z., Pian, Y., Ren, Z., Bi, L., Yuan, Y., Zheng, Y., Jiang, Y., \& Wang, F. (2014). Increased production of suilysin contributes to invasive infection of the Streptococcus suis strain 05ZYH33. Mol Med Rep, 10(6), 2819-2826. doi: 10.3892/mmr.2014.2586.

Hlebowicz, M., Jakubowski, P., \& Smiatacz, T. (2019). Streptococcus suis Meningitis: Epidemiology, Clinical Presentation and Treatment. Vector Borne Zoonotic Dis, 19(8), 557-562. doi: 10.1089/vbz.2018.2399.

Jenkins, A., Diep, B., Mai, T., Vo, N., Warrener, P., Suzich, J., Stover, C., \& Sellman, B. (2015). Differential expression and roles of Staphylococcus aureus virulence determinants during colonization and disease. mBio, 6(1), e02272-02214. doi: 10.1128/mBio.02272-14.

Kouki, A., Haataja, S., Loimaranta, V., Pulliainen, A., Nilsson, U., \& Finne, J. J. T. J. o. b. c. (2011). Identification of a novel streptococcal adhesin P (SadP) protein recognizing galactosyl- $\alpha 1-4$-galactose-containing glycoconjugates: convergent evolution of bacterial pathogens to binding of the same host receptor, 286(45), 38854-38864. doi: 10.1074/jbc.M111.260992.

Kouki, A., Pieters, R., Nilsson, U., Loimaranta, V., Finne, J., \& Haataja, S. J. B. (2013). Bacterial Adhesion of Streptococcus suis to Host Cells and Its Inhibition by 
Carbohydrate Ligands. 2(3), 918-935. doi: 10.3390/biology2030918.

Li, Q., Fu, Y., Ma, C., He, Y., Yu, Y., Du, D., Yao, H., Lu, C., \& Zhang, W. (2017). The non-conserved region of MRP is involved in the virulence of Streptococcus suis serotype 2. Virulence, 8(7), 1274 1289. doi: $10.1080 / 21505594.2017 .1313373$.

Liu, H., Lei, S., Jia, L., Xia, X., Sun, Y., Jiang, H., Zhu, R., Li, S., Qu, G., Gu, J., Sun, C., Feng, X., Han, W., Langford, P. R., \& Lei, L. (2021a). Streptococcus suis serotype 2 enolase interaction with host brain microvascular endothelial cells and RPSA-induced apoptosis lead to loss of BBB integrity. Vet Res, 52(1), 30. doi: 10.1186/s13567-020-00887-6.

Liu, H., Ye, C., Fu, H., Yue, M., Li, X., \& Fang, W. (2021b). Stk and Stp1 participate in Streptococcus suis serotype 2 pathogenesis by regulating capsule thickness and translocation of certain virulence factors. Microbial pathogenesis, 152104607. doi: 10.1016/j.micpath.2020.104607.

Lv, Q., Hao, H., Bi, L., Zheng, Y., Zhou, X., \& Jiang, Y. J. P. (2014). Suilysin remodels the cytoskeletons of human brain microvascular endothelial cells by activating RhoA and Rac1 GTPase. Protein Cell, 5(4), 261-264. doi: 10.1007/s13238-014-0037-0.

Madar Johansson, M., Bélurier, E., Papageorgiou, A., Sundin, A., Rahkila, J., Kallonen, T., Nilsson, U., Maatsola, S., Nyholm, T., Käpylä, J., Corander, J., Leino, R., Finne, J., Teneberg, S., \& Haataja, S. (2020). Streptococcus suisThe binding mechanism of the virulence factor adhesin $\mathrm{P}$ subtype to globotetraosylceramide is associated with systemic disease. J Biol Chem, 295(42), 14305-14324. doi: 10.1074/jbc.RA120.014818.

Musyoki, A. M., Shi, Z., Xuan, C., Lu, G., Qi, J., Gao, F., Zheng, B., Zhang, Q., Li, Y., Haywood, J., Liu, C., Yan, J., Shi, Y., \& Gao, G. F. (2016). Structural and functional analysis of an anchorless fibronectinbinding protein FBPS from Gram-positive bacterium Streptococcus suis. Proc Natl Acad Sci USA, 113(48), 13869-13874. doi: 10.1073/pnas.1608406113.

Pei, X., Liu, M., Zhou, H., \& Fan, H. (2020). Screening for phagocytosis resistance-related genes via a transposon mutant library of Streptococcus suis serotype 2. Virulence, 11(1), 825-838. doi: 10.1080/21505594.2020.1782088.

Seele, J., Tauber, S., Bunkowski, S., Baums, C., ValentinWeigand, P., de Buhr, N., Beineke, A., Iliev, A., Brück, W., \& Nau, R. (2018). The inflammatory response and neuronal injury in Streptococcus suis meningitis. BMC infectious diseases, 18(1), 297. doi: 10.1186/s12879-018-3206-6.

Segura, M. (2020). Streptococcus suis Research: Progress and Challenges. Pathogens, 9(9). doi: 10.3390/pathogens9090707.

Segura, M., Calzas, C., Grenier, D., \& Gottschalk, M. (2016). Initial steps of the pathogenesis of the infection caused by Streptococcus suis: fighting against nonspecific defenses. FEBS Letters, 590(21), 3772-3799. doi:10.1002/1873-3468.12364.
Segura, M., Fittipaldi, N., Calzas, C., \& Gottschalk, M. (2017). Critical Streptococcus suis Virulence Factors: Are They All Really Critical? Trends Microbiol, 25(7), 585-599. doi: 10.1016/j.tim.2017.02.005.

Siegel, S., Reardon, M., \& Ton-That, H. (2017). Anchoring of LPXTG-Like Proteins to the GramPositive Cell Wall Envelope. Current topics in microbiology immunology, 404, 159-175. doi: 10.1007/82_2016_8.

Sun, Q., Li, N., Jia, L., Guo, W., Jiang, H., Liu, B., Bao, C., Liu, M., Huang, J., \& Lei, L. (2020). Ribosomal Protein SA-Positive Neutrophil Elicits Stronger Phagocytosis and Neutrophil Extracellular Trap Formation and Subdues Pro-Inflammatory Cytokine Secretion Against Streptococcus suis Serotype 2 Infection. Frontiers in immunology, 11585399. doi: 10.3389/fimmu.2020.585399.

Tang, Y., Zhang, X., Wu, W., Lu, Z., \& Fang, W. (2012). Inactivation of the sodA gene of Streptococcus suis type 2 encoding superoxide dismutase leads to reduced virulence to mice. Veterinary microbiology, 158, 360-366. doi: 10.1016/j.vetmic.2012.02.028.

Tenenbaum, T., Asmat, T., Seitz, M., Schroten, H., \& Schwerk, C. (2016). Biological activities of suilysin: role in Streptococcus suis pathogenesis. Future microbiology, 11, 941-954. doi: 10.2217/fmb-2016-0028.

Vötsch, D., Willenborg, M., Oelemann, W., Brogden, G., \& Valentin-Weigand, P. (2019). Membrane Binding, Cellular Cholesterol Content and Resealing Capacity Contribute to Epithelial Cell Damage Induced by Suilysin of Streptococcus suis. Pathogens, 9(1). doi: 10.3390/pathogens9010033.

Wang, J., Dong, R., Zou, P., Chen, Y., Li, N., Wang, Y., Zhang, T., \& Pan, X. (2020). Identification of a Novel Linear B Cell Epitope on the Sao Protein of Streptococcus suis Serotype 2. Front Immunol, 111492. doi: 10.3389/fimmu.2020.01492.

Wang, J., Feng, Y., Wang, C., Zheng, F., Hassan, B., Zhi, L., Li, W., Yao, Y., He, E., Jiang, S., \& Tang, J. (2017). Genome-wide analysis of an avirulent strain that induces protective immunity against challenge with virulent Streptococcus suis serotype 2. BMC Microbiol, 17(1), 67. doi: 10.1186/s12866-017-0971-0.

Xia, X., Qin, W., Zhu, H., Wang, X., Jiang, J., \& Hu, J. (2019). How Streptococcus suis serotype 2 attempts to avoid attack by host immune defenses. J Microbiol Immunol Infect, 52(4), 516-525. doi: 10.1016/j.jmii.2019.03.003.

Xie, J., Shen, Z., Anraku, Y., Kataoka, K., \& Chen, X. (2019). Nanomaterial-based blood-brain-barrier (BBB) crossing strategies. Biomaterials, 224, 119491. doi: 10.1016/j.biomaterials.2019.119491.

Yang, C., Chen, B., Zhao, J., Lin, L., Han, L., Pan, S., Fu, L., Jin, M., Chen, H., Zhang, A., \& immunity (2015). TREM-1 signaling promotes host defense during the early stage of infection with highly pathogenic Streptococcus suis. Infection, 83(8), 3293-3301. doi: 10.1128/iai.00440-15.

Zhang, D., Xu, S., Wang, Y., \& Zhu, G. (2021). The Potentials of Melatonin in the Prevention and Treatment of Bacterial Meningitis Disease. Molecules, 26(5), 1419. doi: 10.3390/molecules26051419. 
Zhao, J., Pan, S., Lin, L., Fu, L., Yang, C., Xu, Z., Wei, Y., Jin, M., \& Zhang, A. (2015). Streptococcus suis serotype 2 strains can induce the formation of neutrophil extracellular traps and evade trapping. FEMS Microbiol Lett, 362(6), doi: 10.1093/female/fnv022.
Zhu, J., Xiang, L., Jiang, F., \& Zhang, Z. (2016). Equilibrium of sortase A dimerization on Staphylococcus aureus cell surface mediates its cell wall sorting activity. Experimental biology medicine, 241(1), 90-100. doi: 10.1177/1535370215592122. 